\title{
The Rules of Entrainment: Are CA1 Gamma Oscillations Externally Imposed or Locally Governed?
}

\author{
Antonio Fernández-Ruiz ${ }^{1,2 *}$ and Erik W. Schomburg ${ }^{1,3 *}$ \\ ${ }^{1}$ Neuroscience Institute, Langone Medical Center, New York University, New York, New York 10016, ${ }^{2}$ School of Physics, Complutense University of Madrid, \\ 28040 Madrid, Spain, and ${ }^{3}$ Physics Department, California Institute of Technology, Pasadena, California 91125 \\ Review of Zemankovics et al.
}

Gamma oscillations $(\sim 30-90 \mathrm{~Hz})$ in the local field potential (LFP) are a widespread signature of information processing in neural circuits and have been linked to cognitive functions ranging from sensory perception and attention to memory encoding and the organization of neuronal assemblies (Engel et al., 2001; Buzsáki and Wang, 2012). Special emphasis has been placed on the gamma-band phasesynchronization among different brain areas, which has been proposed as a mechanism to bind together information processed in distant regions into a unified representation (Engel et al., 2001) or to coordinate different networks engaged in a common memory task (Montgomery and Buzsáki, 2007).

Despite intensive study, the cellular mechanisms and functional roles of gamma oscillations and synchronization remain unclear in most cases (Buzsáki and Wang, 2012). In the case of LFP oscillations, there are two main questions to be answered: (1) what currents generate the

Received Sept. 27, 2013; revised 0ct. 29, 2013; accepted Nov. 1, 2013.

We thank G. Buzsáki for helpful comments on the manuscript. We also thank European Molecular Biology Organization, National Institutes of Health and Human Frontiers Science Program for generous support.

The authors declare no conflict of interest.

${ }^{*}$ A.F.-R. and E.W.S. contributed equally to this work

Correspondence should be addressed to either Antonio Fernández-

Ruiz or Erik W. Schomburg, NYU Neuroscience Institute, Langone Medical Center, New York University, East River Science Park, 450 East 29th Street, 9th Floor, New York, NY 10016. E-mail: antferrui@gmail.com or eschomburg@gmail.com.

DOI:10.1523/JNEUROSCI.4151-13.2013

Copyright $\odot 2013$ the authors $\quad 0270-6474 / 13 / 3319045-03 \$ 15.00 / 0$
LFP, and (2) what network interactions coordinate the rhythmic activity? Both questions must be addressed to gain a proper understanding of the physiological mechanisms of gamma-band LFP oscillations. For example, does a particular gamma signal reflect the rhythmic activation of afferent synapses, or does it emerge as a result of local circuit computations (or both)?

An extensively studied example of gamma oscillations are those recorded in the rodent hippocampal CA1 area, which are dynamically organized within ongoing theta oscillations during active exploration and rapid eye movement (REM) sleep. Different origins and mechanisms have been proposed for these oscillations, including excitatory inputs from area CA3 and entorhinal cortex (EC), as well as locally coordinated inhibitory perisomatic currents (Csicsvari et al., 2003). There are likely to be multiple processes at play, especially given the different characteristics of LFPs and spiking occurring at different phases of the theta cycle within the same region (Colgin et al., 2009; Belluscio et al., 2012).

Cholinergic agonists such as carbachol (CCh) have been shown to induce oscillations in CA3 in hippocampal slices resembling in vivo gamma oscillations (Fisahn et al., 1998). How these oscillations propagate to target regions is not fully understood, and this was the focus of a study by Zemankovics and collaborators (2013). Working with mouse hippocampal slices, they found that CA1 LFPs during CChinduced oscillations primarily reflect perisomatic inhibitory currents onto CA1 pyramidal cells (PCs) driven by rhythmic excitatory input from CA3 PCs onto CA1 interneurons. Firing of CA1 interneurons was strongly modulated by phasic CA 3 excitation, but CA1 pyramidal cells were only weakly modulated. Furthermore, the PCs tended to fire at an earlier phase of the oscillation, shortly before the incoming excitation from CA3. So what controls the firing of CA1 PCs? In contrast with interneurons, the dominant phasic currents in PCs were inhibitory. Moreover, the preferred firing phase of PCs aligned with that of interneurons after infusion of gabazine (a $\mathrm{GABA}_{\mathrm{A}}$ blocker) into $\mathrm{CA} 1$ stratum pyramidale. These data suggest that phasic input from CA3 excites both CA1 PCs and interneurons, but because interneurons have lower spike thresholds and more electrotonically compact dendrites than PCs, they respond more quickly and reliably to the CA3 input and suppress the concurrent excitation of CA1 PCs. PCs are therefore most likely to fire at the point in the cycle at which they are least inhibited, shortly before $(\sim 6 \mathrm{~ms})$ the interneurons fire maximally.

These results can be incorporated into various models of hippocampal function that emphasize the role of oscillations mediating input integration and cell assembly formation (Buzsáki, 2010). Rather than directly entraining CA1 PCs, CA3 gamma oscillations may recruit feedfor- 
ward inhibition to create temporal windows of opportunity for CA1 PCs to fire. Longer time scale mechanisms, such as NMDA-mediated currents and dendritic calcium events, could conceivably aid in this process, as well as engage synaptic plasticity mechanisms to modify the network at the levels of both afferent CA3 terminals and the local interneuron plexus (Spruston, 2008). By engaging local interneurons in another layer of computation to selectively activate discrete assemblies of PCs, gamma oscillations may thus enhance the information processing capabilities of CA1 (Csicsvari et al., 2003; Buzsáki and Wang, 2010). Furthermore, the temporal clustering of CA1 PC spikes may increase the efficacy of the activated assemblies on their downstream targets (Buzsáki, 2010).

The key question, as always, is how these in vitro findings relate to in vivo activity in the behaving animal. LFPs are notoriously difficult to interpret; they are a complex mixture of synaptic inputs and local activity in the recorded region, and they can be strongly distorted by volume conduction. In the case of gamma-band signals at the CA1 pyramidal layer, perisomatic synaptic currents onto CA1 PCs, passive return currents from dendritic synapses, volume-conducted potentials (especially from very strong gamma currents originating in the dentate gyrus), and spectral contamination from synchronized action potentials (Belluscio et al., 2012) can all generate substantial power at gamma frequencies in the same location. Differentiating these influences is both a challenge and a necessary step for understanding the origin and function of gamma oscillations.

The observation by Zemankovics et al. (2013) that the interneurons recruited by the gamma input can effectively suppress the CA3 excitation of most CA1 PCs is consistent with the reduced PC firing during the theta phases of maximal EC and CA3 input while the animal is running (Mizuseki et al., 2009). Indeed, it has been shown that CA3 input directly evokes CA1 PC spikes in a sparse manner (Fernández-Ruiz et al., 2012). Zemankovics et al. (2013) propose that the weak coupling between CA1 PCs and CA3 gamma oscillations might enable CA1 cells to respond efficiently to excitatory input from the EC. The properly timed combination of excitation from the Schaffer collaterals (CA3) and the temporo-ammonic pathway (EC) has been shown to evoke active dendritic currents in PCs, boosting and prolonging the impact of synaptic poten- tials at the cell somata (Takahashi and Magee, 2009). Such slow dendritic plateau potentials may also allow PC assemblies to be organized and timed by local network mechanisms. It is plausible that several multiplexed mechanisms coexist in vivo for information transfer between these regions, subject to the behavioral state and processing demands.

One caveat to the Zemankovics et al. (2013) conclusions is the unknown similarity of CCh-induced gamma activity to the endogenous gamma activity occurring in behaving animals. Various gamma-band patterns have been reported in the hippocampus in vivo depending on behavior (Montgomery and Buzsáki, 2007; Belluscio et al., 2012), and in vitro, depending on the activated receptors (Pálhalmi et al., 2004). For the CA1 gamma LFPs induced by CA3 input, it is likely that similar mechanisms are involved in both cases, but this remains to be carefully examined, and it is unclear which mechanisms may dominate during natural brain activity when simultaneous activation of multiple inputs is present. For instance, Pálhalmi et al. (2004) hypothesized that differences in spectral and pharmacological characteristics of cholinergically-induced gamma and metabotrobic glutamate receptor-induced gamma in CA3 may be explained by differences in interneuron participation. This could plausibly be the case in CA1 as well, depending on the response of the CA1 network to the spatiotemporal motif of CA3 input. Furthermore, EC input activates a different subset of interneurons (Klausberger and Somogyi, 2008) and may therefore engage different mechanisms.

Finally, Zemankovics et al. (2013) could not further differentiate the roles of several subclasses of interneurons, such as basket, axo-axonic (chandelier), or bistratified cells, all of which fell under the PV + category. Bistratified cells are the most strongly modulated by in vivo gamma oscillations in CA1 of anesthetized rats (Tukker et al., 2007), and CCK + interneurons have recently been implicated in underlying strong feedforward inhibition from CA3 to CA1 (Basu et al., 2013). Although these dendrite-targeting interneurons are surely important for regulating the excitation of $\mathrm{PCs}$, they might be less effective at precisely controlling spike timing compared with perisomatically targeting interneurons (Royer et al., 2012). In addition, they have characteristically different firing patterns during theta, gamma, sharp wave-ripples, etc. (Klausberger and Somogyi, 2008), and different hypothesized roles in modulating the gain and timing of PCs. Thus, further in vitro and in vivo exper- iments using techniques that can identify interneuron subtypes are needed to clarify their functional role during hippocampal computations.

The literature has defined gamma oscillations over a wide spectral band (sometimes inconsistently). It is likely that different activity patterns are spectrally overlapped, spatially intermingled, and temporally coordinated by concurrent rhythms in a state-dependent manner. Lumping these different patterns together into a generic "gamma oscillation" may obscure their roles in the precise organization of the hippocampal network and impede efforts to understand the underlying cellular mechanisms. In future work, it will therefore be important to dissect the different network phenomena contributing to gamma-band LFPs in a given region. Appropriate spatiotemporal sampling of LFPs and unit activity in different behavioral conditions, as well as accurate analytical tools for studying their ongoing dynamics, is necessary. Understanding the roles of the diverse array of local interneurons in mediating these complex interactions is one of the most exciting lines of research currently underway in systems neuroscience, and further efforts toward this end will surely continue to uncover the breathtaking sophistication of network processing in cortical circuits. The work by Zemankovics et al. (2013) is an important step in this direction.

\section{References}

Basu J, Srinivas KV, Cheung SK, Taniguchi H, Huang ZJ, Siegelbaum SA (2013) A corticohippocampal learning rule shapes inhibitory microcircuit activity to enhance hippocampal information flow. Neuron 79:1208-1221. CrossRef Medline

Belluscio MA, Mizuseki K, Schmidt R, Kempter R, Buzsáki G (2012) Cross-frequency phasephase coupling between theta and gamma oscillations in the hippocampus. J Neurosci 32: 423-435. CrossRef Medline

Buzsáki G (2010) Neural syntax: cell assemblies, synapsembles, and readers. Neuron 68:362385. CrossRef Medline

Buzsáki G, Wang XJ (2012) Mechanisms of gamma oscillations. Annu Rev Neurosci 35: 203-225. CrossRef Medline

Colgin LL, Denninger T, Fyhn M, Hafting T, Bonnevie T, Jensen O, Moser MB, Moser EI (2009) Frequency of gamma oscillations routes flow of information in the hippocampus. Nature 462:353-357. CrossRef Medline

Csicsvari J, Jamieson B, Wise KD, Buzsáki G (2003) Mechanisms of gamma oscillations in the hippocampus of the behaving rat. Neuron 37:311-322. CrossRef Medline

Engel AK, Fries P, Singer W (2001) Dynamic preditions: oscillation and synchrony in top- 
down processing. Nat Rev Neurosci 2:704716. CrossRef Medline

Fernández-Ruiz A, Makarov VA, Benito N, Herreras O (2012) Schaffer-specific local field potentials reflect discrete excitatory events at gamma frequency that may fire postsynaptic hippocampal CA1 units. J Neurosci 32:51655176. CrossRef Medline

Fisahn A, Pike FG, Buhl EH, Paulsen O (1998) Cholinergic induction of network oscillations at $40 \mathrm{~Hz}$ in the hippocampus in vitro. Nature 394:186-189. CrossRef Medline

Klausberger T, Somogyi P (2008) Neuronal diversity and temporal dynamics: the unity of hippocampal circuit operations. Science 321: 53-57. CrossRef Medline

Mizuseki K, Sirota A, Pastalkova E, Buzsáki G (2009) Theta oscillations provide temporal windows for local circuit computation in the entorhinal-hippocampal loop. Neuron 64: 267-280. CrossRef Medline

Montgomery SM, Buzsáki G (2007) Gamma oscillations dynamically couple hippocampal $\mathrm{CA} 3$ and CA1 regions during memory task performance. Proc Natl Acad Sci U S A 104: 14495-14500. CrossRef Medline

Pálhalmi J, Paulsen O, Freund TF, Hájos N (2004) Distinct properties of carbachol- and DHPG-induced network oscillations in hippocampal slices. Neuropharmacology 47: 381-389. CrossRef Medline

Royer S, Zemelman BV, Losonczy A, Kim J, Chance F, Magee JC, Buzsáki G (2012) Control of timing, rate and bursts of hippocampal place cells by dendritic and somatic inhibition. Nat Neurosci 15:769-775. CrossRef Medline
Spruston N (2008) Pyramidal neurons: dendritic structure and synaptic integration. Nat Rev Neurosci 9:206-221. CrossRef Medline

Takahashi H, Magee JC (2009) Pathway interactions and synaptic plasticity in the dendritic tuft regions of CAl pyramidal neurons. Neuron 62:102-111. CrossRef Medline

Tukker JJ, Fuentealba P, Hartwich K, Somogyi P, Klausberger T (2007) Cell type-specific tuning of hippocampal interneuron firing during gamma oscillations in vivo. J Neurosci 27 : 8184-8189. CrossRef Medline

Zemankovics R, Veres JM, Oren I, Hájos N (2013) Feedforward inhibition underlies the propagation of cholinergically induced gamma oscillations from hippocampal CA3 to CA1. J Neurosci 33:12337-12351. CrossRef Medline 